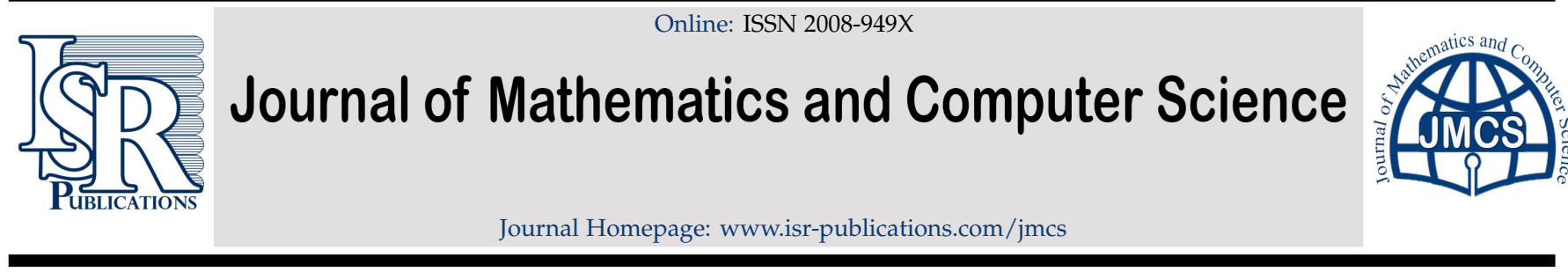

\title{
Kamenev type oscillation criteria for second order impulsive differential equations
}

\author{
S. Euat Tallah, ${ }^{\mathrm{a}, *}$ M. M. A. Elsheikh ${ }^{\mathrm{b}}$, G. A. F. Ismail ${ }^{\mathrm{a}}$
}

${ }^{a}$ Department of Mathematics, University College for women, Ain shams university, Cairo, Egypt.

${ }^{b}$ Department of Mathematics and computer science, Faculty of science, Menoufia university, Shebin El-Koom, Egypt.

\begin{abstract}
The oscillation of second order impulsive differential equations is discussed using Riccati transformations technique. New oscillation criteria are established, to improve and extend some recent results in the literature. Two illustrative examples are given.
\end{abstract}

Keywords: Kameneve type oscillation, damping terms, impulsive, Riccati transformations.

2020 MSC: 34A37, 34C10, 34C29.

(C)2022 All rights reserved.

\section{Introduction}

In this paper, we consider the second order impulsive Sturm-Liouville differential equations

$$
\begin{aligned}
{\left[\left(\mathrm{r}(\mathrm{t}) \mathrm{x}^{\prime}(\mathrm{t})\right]^{\prime}+\mathrm{p}(\mathrm{t}) \mathrm{x}^{\prime}(\mathrm{t})+\mathrm{q}(\mathrm{t}) \mathrm{x}(\mathrm{t})\right.} & =0, \mathrm{t} \geqslant \mathrm{t}_{0}, \mathrm{t} \neq \mathrm{t}_{\mathrm{k}} ; \\
\Delta\left[\left(\mathrm{r}\left(\mathrm{t}_{\mathrm{k}}\right) x^{\prime}\left(\mathrm{t}_{\mathrm{k}}\right)\right]+\mathrm{b}_{\mathrm{k}} x\left(\mathrm{t}_{\mathrm{k}}\right)\right. & =0,
\end{aligned}
$$

the more general impulsive differential equations

$$
\begin{aligned}
{\left[\left(r(t) \Psi(x(t)) x^{\prime}(t)\right]^{\prime}+p(t) x^{\prime}(t)+q(t) x(t)\right.} & =0, t \geqslant t_{0}, t \neq t_{k} \\
\Delta\left[\left(r\left(t_{k}\right) \Psi\left(x\left(t_{k}\right)\right) x^{\prime}\left(t_{k}\right)\right]+b_{k} x\left(t_{k}\right)\right. & =0
\end{aligned}
$$

and

$$
\begin{gathered}
{\left[r(t) \Psi(x(t))\left(x^{\prime}(t)\right)^{\gamma}\right]^{\prime}+p(t)\left(x^{\prime}(t)\right)^{\gamma}+q(t) f(x(t))=0 \text { for } t \geqslant t_{0}, t \neq t_{k} ;} \\
\Delta\left[r\left(t_{k}\right) \Psi\left(x\left(t_{k}\right)\right)\left(x^{\prime}\left(t_{k}\right)\right)^{\gamma}\right]^{\prime}+b_{k} f\left(x\left(t_{k}\right)\right)=0,
\end{gathered}
$$

where $p(t), q(t)$ are left piecewise continuous on $\left[t_{0}, \infty\right) ; \Psi(x(t))$ and $r(t)$ are continuous and positive for some $t_{0}>0$. The function $\Psi(x(t))$ satisfies $0<\zeta(t) \leqslant \Psi(x(t)) \leqslant \eta(t)$; where $\gamma \geqslant 1$ is a ratio of odd positive integers, the function $f(x(t))$ satisfies $x f(x)>0$ for $x \neq 0, f(x) / x^{\gamma} \geqslant \mu$, for $\mu>0$ and

\footnotetext{
*samah_saad75@yahoo.com

Email addresses: samah_saad75@yahoo.com (S. Euat Tallah), msheikh_1999@yahoo.com (M. M. A. Elsheikh),

gam_ismail@yahoo.com (G. A. F. Ismail)
}

doi: $10.22436 /$ jmcs.026.02.07

Received: 2021-06-24 Revised: 2021-08-17 Accepted: 2021-09-17 
for all $x \neq 0$ and $N$ is a set of positive integers. The sequence of times $\left\{t_{k}\right\}_{k \in N}$ is strictly increasing with $t_{k} \geqslant t_{0}$ and $\lim _{k \rightarrow \infty} t_{k}=\infty ; \Delta z\left(t_{k}\right)=z\left(t_{k}^{+}\right)-z\left(t_{k}^{-}\right)$; and the coefficient $\left\{b_{k}\right\}$ is a sequence of real numbers. A nontrivial solution of the differential equation is called oscillatory if it has arbitrarily large zeros, otherwise, it is called nonoscillatory. The equation is said to be oscillatory if all its nontrivial solutions are oscillatory.

In the last few decades the oscillatory behavior of solutions of second-order differential equations has received great interest in the literature due to its importance in a wide variety of applications in physics, mathematics, engineering and biology, etc. (see $[1,3,4,7-12,14,15,17,19,21,26,28]$ ). Recently, a lot of works have been appeared in the literature in the area of impulsive differential equations $[5,6,22,25]$. Although the solutions of ordinary differential equations are continuously differentiable, however, in some cases the impulsive differential equations generally possess non-continuous solutions. Eq. (1.1) can be considered as an equation of motion in which the movement speed of the mass point changes discontinuously by abrupt changes at certain moments. (See also [20,24] for models from mathematical biology where impulsive-type phenomena are idealized by external sources of evolutive PDE's.) In dynamical models formulated by partial differential equations, impulsive-type effects are often modeled by external sources perturbing the natural evolution of the related models (see [16]). Very recently, Wen et al. [25] gave Philos-type oscillation criteria for the damped second order linear impulsive differential equation

$$
\begin{aligned}
x^{\prime \prime}(t)+p(t) x^{\prime}(t)+q(t) x(t) & =0, t \geqslant t_{0}, t \neq t_{k} ; \\
\Delta\left(x^{\prime}\left(t_{k}\right)\right)+b_{k} x\left(t_{k}\right) & =0 .
\end{aligned}
$$

In the case, $b_{k}=0$ for all $k \in N$, the oscillation is caused by the effect of damping only and Eq. (1.4) becomes:

$$
x^{\prime \prime}(\mathrm{t})+\mathrm{p}(\mathrm{t}) x^{\prime}(\mathrm{t})+\mathrm{q}(\mathrm{t}) x(\mathrm{t})=0 .
$$

Many authors have studied this [2, 27] (and references cited in). Meanwhile, Sugie et al. [23] discussed the effect of impulses on the oscillation of second-order linear differential equations. They proved that the oscillation of mass point could be caused by the effect of impulses only, while if it does not exist, the mass point does not oscillate. They discussed the undamped case in which the function $p(t)=0$, i.e., the differential equations

$$
x^{\prime \prime}(\mathrm{t})+\mathrm{q}(\mathrm{t}) \mathrm{x}(\mathrm{t})=0, \mathrm{t} \geqslant \mathrm{t}_{0}, \mathrm{t} \neq \mathrm{t}_{\mathrm{k}} ; \quad \Delta\left(\mathrm{x}^{\prime}\left(\mathrm{t}_{\mathrm{k}}\right)\right)+\mathrm{b}_{\mathrm{k}} \mathrm{x}\left(\mathrm{t}_{\mathrm{k}}\right)=0 .
$$

Sugie [22] discussed the oscillation of the second order self-adjoint impulsive differential equations of the type,

$$
\left[\left(r(t) x^{\prime}(t)\right]^{\prime}+q(t) x(t)=0, t \geqslant t_{0}, t \neq t_{k} ; \quad \Delta\left[\left(r\left(t_{k}\right) x^{\prime}\left(t_{k}\right)\right]+b_{k} x\left(t_{k}\right)=0,\right.\right.
$$

to extend previous results of Kamenev-Philos type oscillation. Moreover, he proved that the differential equation (1.5) may oscillate even if the total impulse is small.

In [13], He and Ge established sufficient conditions for the oscillation of the solutions of the impulsive differential equation

$$
\begin{aligned}
{\left[r(t)\left(x^{\prime}(t)\right)^{\gamma}\right]^{\prime}+f(t, x(t)) } & =0 \text { for } t \geqslant t_{0}, t \neq t_{k} ; \\
x\left(t_{k}^{+}\right) & =g_{k}\left(x\left(t_{k}\right)\right), \\
x^{\prime}\left(t_{k}^{+}\right) & =\epsilon_{k} x^{\prime}\left(t_{k}\right), \text { for } k=1,2, \ldots
\end{aligned}
$$

This paper aims to discuss Philos-Kamenev type oscillation for a class of second-order linear impulsive Sturm differential equations with damping (1.1), a class of second-order nonlinear impulsive differential equations with damping (1.2), and a class of second-order half-linear impulsive differential equations with damping Eq. (1.3). First, in Section 2, we investigate the oscillatory behavior of the solution of Eq. 
(1.1), which the damping or the impulses can evaluate. Then, in Section 3, we discuss the oscillation of the more general Eq. (1.2). Our results improve and extend the recent results of [22, 25].

Also, we establish oscillation results more general than those obtained in [13], for the second-order half-linear impulsive differential Eq. (1.3) with a different method. Finally, in Section 4, we give two illustrative examples.

Definition 1.1 ([25]).

1) Let $t_{k}$ be the impulsive moments for each $k \in N$ and let $N(t)$ be the number of the moments of impulsive effect in the interval $\left[t_{0}, t\right)$. Then $N(t)=0$ for $t_{0} \leqslant t \leqslant t_{1}, N(t)=k$ for $t_{k} \leqslant t \leqslant t_{k+1}$.

2) A function $x$ is said to be a solution of Eqs. (1.1)-(1.3) on $\left[t_{0}, \infty\right)$ if

a) $x(t)$ is continuous on $\left[t_{0}, \infty\right)$;

b) $x(t)$ satisfies the first equality of Eqs. (1.1)-(1.2) on $\left[t_{0}, \infty\right) \backslash\left\{t_{k}, k \in N\right\}$;

c) $x^{\prime}(t)$ has two-sided limits and left continuous at points $t_{k}, x^{\prime}(t)$ satisfies the second equality of Eqs. (1.1)-(1.3).

Suppose that $D=\left\{(t, s): t_{0} \leqslant s \leqslant t \leqslant \infty\right\}$ and $D_{0}=\left\{(t, s): t_{0} \leqslant s<t \leqslant \infty\right\}$. We say that $H \in C(D,[0, \infty))$ belongs to the class $\omega_{\gamma}$ if it satisfies the conditions

(i) $H(t, t)=0$ for $t \geqslant t_{0}, H(t, s)>0$ for all $(t, s) \in D_{0}$;

(ii) $\partial H / \partial s=-h(t, s)(H(t, s))^{\frac{\gamma}{\gamma+1}}$ for all $(t, s) \in D$.

The following lemma will be needed in the proof of our results.

Lemma 1.2 ([18]). Let $\gamma \geqslant 1$ be a ratio of two odd numbers. Then

$$
A^{1+\frac{1}{\gamma}}-(A-B)^{1+\frac{1}{\gamma}} \leqslant \frac{B^{\frac{1}{\gamma}}}{\gamma}[(\gamma+1) A-B], \quad C^{\frac{\gamma+1}{\gamma}}-\frac{\gamma+1}{\gamma} C^{\frac{1}{\gamma}} \geqslant D^{\frac{\gamma+1}{\gamma}} .
$$

\section{Main results}

Theorem 2.1. If there exists a function $\mathrm{H}(\mathrm{t}, \mathrm{s}) \in \mathrm{D}$ satisfying the conditions (i) and (ii) and moreover

$$
\begin{aligned}
\limsup _{t \rightarrow \infty} \frac{1}{H\left(t, t_{0}\right)} & \times\left\{\sum_{k=1}^{N(t)} H\left(t, t_{k}\right) b_{k}+\int_{t_{0}}^{t} H(t, s) q(s)-\frac{1}{4}\left[r(s) h^{2}(t, s)\right.\right. \\
& \left.\left.+\frac{p^{2}(s) H(t, s)}{r(s)}+2 h(t, s) p(s) \sqrt{H(t, s)}\right] d s\right\}=\infty,
\end{aligned}
$$

then Eq. (1.1) is oscillatory.

Proof. Let $x(t)$ be a non-oscillatory solution of Eq. (1.1). Without loss of generality, we may assume that $x(t) \neq 0$ for all $T \geqslant t_{0}$. Let

$$
u(t)=\frac{r(t) x^{\prime}(t)}{x(t)}
$$

Then by (1.1) for all $t \geqslant t_{0}$, we have

$$
u^{\prime}(t)=-q(t)-\frac{u^{2}(t)}{r(t)}-p \frac{u(t)}{r(t)}, \quad \text { for } \quad t \neq t_{k} .
$$

Since $x(t)$ is continuous on $[T, \infty)$, then

$$
\Delta u\left(t_{k}\right)=u\left(t_{k}^{+}\right)-u\left(t_{k}^{-}\right)=\frac{r\left(t_{k}^{+}\right) x^{\prime}\left(t_{k}^{+}\right)}{x\left(t_{k}^{+}\right)}-\frac{r\left(t_{k}^{-}\right) x^{\prime}\left(t_{k}^{-}\right)}{x\left(t_{k}^{-}\right)}=\frac{\Delta\left[r\left(t_{k}\right) x^{\prime}\left(t_{k}\right)\right]}{x\left(t_{k}\right)}=-b_{k} .
$$


Therefore, the function $u(t)$ satisfies

$$
u^{\prime}(t)+q(t)+\frac{u^{2}(t)}{r(t)}+p(t) \frac{u(t)}{r(t)}=0, \text { for } \quad t \neq t_{k}, \quad \Delta u\left(t_{k}\right)+b_{k}=0
$$

Following [25], we assume $t_{m-1} \leqslant T<t_{m}$ for a positive integer $m$. For sufficiently large $t$, we take a positive integer $n=j(t)$ such that, $t_{n} \leqslant t<t_{n+1}$. Let I denote the interval $[T, t]$ except the points $t_{m}, t_{m+1}, t_{m+2}, \ldots, t_{n}$. Multiplying the first equation of (2.2) by $H(t, s)$ and integrating from $T$ to $t$, we get

$$
\int_{T}^{t} q(s) H(t, s) d s=\int_{I} q(s) H(t, s) d s=-\int_{I} H(t, s) u^{\prime}(s) d s-\int_{T}^{t} H(t, s) \frac{u^{2}(s)}{r(s)} d s-\int_{T}^{t} H(t, s) p(s) \frac{u(s)}{r(s)} d s,
$$
i.e.,

$$
\begin{aligned}
\int_{T}^{t} q(s) H(t, s) d s= & -\int_{T}^{t_{m}} H(t, s) u^{\prime}(s) d s-\sum_{k=m}^{n-1} \int_{t_{k}}^{t_{k+1}} H(t, s) u^{\prime}(s) d s-\int_{t n}^{t} H(t, s) u^{\prime}(s) d s \\
& -\int_{T}^{t}\left[H(t, s) \frac{u^{2}(s)}{r(s)}-H(t, s) p(s) \frac{u(s)}{r(s)}\right] d s .
\end{aligned}
$$

Using integration by parts and the continuity of $H(t, s)$ on $D$, we get

$$
-\int_{T}^{t_{m}} H(t, s) u^{\prime}(s) d s=\sum_{k=m}^{n-1}\left[H\left(t, t_{k}\right) u\left(t_{k}^{+}\right)-H\left(t, t_{k+1}\right) u\left(t_{k+1}^{-}\right)\right]+\sum_{k=m}^{n-1} \int_{t_{k}}^{t_{k+1}} u(s) \frac{\partial H(t, s)}{\partial s} d s .
$$

Since $H(t, t)=0$, then

$$
\int_{t_{n}}^{t} H(t, s) u^{\prime}(s) d s=H\left(t, t_{n}\right) u\left(t_{n}^{+}\right)+\int_{t_{n}}^{t} u(s) \frac{\partial H(t, s)}{\partial s} d s
$$

Going through as in [22], using the difference operator

$$
\begin{aligned}
\int_{T}^{t} q(t) H(t, s) d s= & H(t, T) u(T)-\int_{T}^{t} H(t, s)\left[\frac{u^{2}(s)}{r(s)}+p(s) \frac{u(s)}{r(s)}\right] d s \\
& \left.+\sum_{k=m}^{n} H\left(t, t_{k}\right) \triangle u\left(t_{i}\right)-\int_{T}^{t} u(s) h(t, s) \sqrt{H(t, s}\right) d s \\
= & H(t, T) u(T)-\int_{T}^{t} H(t, s)\left[\frac{u^{2}(s)}{r(s)}+p(s) \frac{u(s)}{r(s)}\right] d s \\
& \left.-\sum_{k=m}^{N(t)} H\left(t, t_{k}\right) b_{k}-\int_{T}^{t} u(s) h(t, s) \sqrt{H(t, s}\right) d s .
\end{aligned}
$$

By completing the squares, we get

$$
\begin{aligned}
\int_{T}^{t} q(t) H(t, s) d s= & H(t, T) u(T)-\int_{I}\left\{u(s) \sqrt{\frac{H(t, s)}{r(s)}}+\sqrt{\frac{r(s)}{4 H(t, s)}}\left(\frac{H(t, s) p(s)}{r(s)}+h(t, s) \sqrt{H(t, s)}\right\}^{2} d s\right. \\
& +\frac{1}{4} \int_{I} \frac{r(s)}{H(t, s)}\left(\frac{H(t, s) p(s)}{r(s)}+h(t, s) \sqrt{H(t, s)}\right)^{2} d s-\sum_{k=1}^{N(t)} H\left(t, t_{k}\right) b_{k} \\
\leqslant & H(t, T) u(T)+\frac{1}{4} \int_{I} \frac{r(s)}{H(t, s)}\left(\frac{H(t, s) p(s)}{r(s)}+h(t, s) \sqrt{H(t, s)}\right)^{2} d s-\sum_{k=1}^{N(t)} H\left(t, t_{k}\right) b_{k} .
\end{aligned}
$$


Then

$$
\int_{T}^{t} q(t) H(t, s) d s-\frac{1}{4} \int_{I} \frac{r(s)}{H(t, s)}\left(\frac{H(t, s) p(s)}{r(s)}+h(t, s) \sqrt{H(t, s)}\right)^{2} d s+\sum_{k=1}^{N(t)} H\left(t, t_{k}\right) b_{k} \leqslant H(t, T) u(T) .
$$

\section{Moreover}

$$
\begin{aligned}
\int_{t_{0}}^{t}\left[q(t) H(t, s)-\frac{1}{4} \frac{r(s)}{H(t, s)}\left(\frac{H(t, s) p(s)}{r(s)}+h(t, s) \sqrt{H(t, s)}\right)^{2}\right] d s+\sum_{k=1}^{N(t)} H\left(t, t_{k}\right) b_{k} \\
=\int_{t_{0}}^{T} q(t) H(t, s) d s+\sum_{k=1}^{m-1} H\left(t, t_{k}\right) b_{k}-\frac{1}{4} \int_{t_{0}}^{T} \frac{r(s)}{H(t, s)}\left(\frac{H(t, s) p(s)}{r(s)}+h(t, s) \sqrt{H(t, s)}\right)^{2} d s \\
\quad+H(t, T) u(T)+\int_{T}^{t} q(t) H(t, s) d s+\sum_{k=m}^{N} H\left(t, t_{k}\right) b_{k}-\frac{1}{4} \int_{T}^{t} \frac{r(s)}{H(t, s)}\left(\frac{H(t, s) p(s)}{r(s)}+h(t, s) \sqrt{H(t, s)}\right)^{2} d s \\
\leqslant \int_{t_{0}}^{T} q(t) H(t, s) d s+H(t, T) u(T)+\sum_{k=1}^{m-1} H\left(t, t_{k}\right) b_{k}-\frac{1}{4} \int_{t_{0}}^{T} \frac{r(s)}{H(t, s)}\left(\frac{H(t, s) p(s)}{r(s)}+h(t, s) \sqrt{H(t, s)}\right)^{2} d s .
\end{aligned}
$$

Since $p(t)>0$ and $\frac{\partial H}{\partial s}=-h(t, s) \sqrt{H(t, s)}$, then we obtain

$$
\begin{aligned}
& \int_{t_{0}}^{t} q(t) H(t, s) d s-\frac{1}{4} \int_{t_{0}}^{t} \frac{r(s) p(s)}{H(t, s)}\left(\frac{H(t, s)}{r(s)}+\frac{h(t, s) \sqrt{H(t, s)}}{p(s)}\right)^{2} d s+\sum_{k=1}^{N(t)} H\left(t, t_{k}\right) b_{k} \\
& \leqslant \int_{t_{0}}^{T} q(t) H(t, s) d s+H(t, T) u(T)+\sum_{k=1}^{m-1} H\left(t, t_{k}\right) b_{k} \\
& \leqslant \int_{t_{0}}^{T}|q(t)| H(t, s) d s+H(t, T)|u(T)|+\sum_{k=1}^{m-1} H\left(t, t_{k}\right)\left|b_{k}\right| \leqslant H\left(t, t_{0}\right)\left[\int_{t_{0}}^{T}|q(t)| d s+|u(T)|+\sum_{k=1}^{m-1}\left|b_{k}\right|,\right.
\end{aligned}
$$

i.e.,

$$
\begin{aligned}
\frac{1}{H\left(t, t_{0}\right)} & \times\left\{\sum_{k=1}^{N(t)} H\left(t, t_{k}\right) b_{k}+\int_{t_{0}}^{t} H(t, s) q(s)-\frac{1}{4}\left[r(s) h^{2}(t, s)+\frac{p^{2}(s) H(t, s)}{r(s)}+2 h(t, s) p(s) \sqrt{H(t, s)}\right] d s\right\} \\
& \leqslant \int_{t_{0}}^{T}|q(t)| d s+|u(T)|+\sum_{k=1}^{m-1}\left|b_{k}\right|<\infty .
\end{aligned}
$$

This contradicts the condition (2.1) and the proof is completed.

Remark 2.2. In the special case $r(t)=1$, the criterion (2.1) includes condition (8) of [25]. Moreover in the case $p(t)=0,(2.1)$ includes the criterion (3.1) of [22].

Theorem 2.3. Suppose that for $\alpha>1,0 \leqslant \beta<1$,

$$
\limsup _{t \rightarrow \infty} \frac{1}{t^{\alpha}} \times\left\{\int_{t_{0}}^{t}(t-s)^{\alpha} s^{\beta} q(s) d s+\sum_{k=1}^{N(t)}\left(t-t_{k}\right)^{\alpha} t_{k}^{\beta} b_{k}\right\}=\infty,
$$

and

$$
\limsup _{t \rightarrow \infty} \frac{1}{t^{\alpha}} \times\left\{\int_{t_{0}}^{t} r(s)(t-s)^{\alpha-2} s^{\beta-2}\left[(t-s) \frac{s p(s)}{r(s)}+\alpha s-\beta(t-s)\right]^{2} d s\right\}<\infty .
$$

Then Eq. (1.1) is oscillatory.

Proof. The proof is similar to that of Theorem 2 in [25], and so it is omitted.

Remark 2.4. In the special case $r(t)=1$, Theorem 2.3 includes Theorem 2 of [25] and Corollary 2 of [22]. 


\section{Philos-Kamenev-type oscillation results}

In this section, we discuss the oscillation of the more general second-order damped impulsive differential Eq. (1.2) and the second-order half-linear damped impulsive differential Eq. (1.3).

Theorem 3.1. Suppose that there exists a continuously differentiable function $\rho(t)$ on $\left[t_{0}, \infty\right)$. If

$$
\begin{aligned}
\limsup _{t \rightarrow \infty} \frac{1}{H\left(t, t_{0}\right)} \times\left\{\int_{t_{0}}^{t} \varphi(s) H(t, s) d s-\frac{1}{4} \int_{t_{0}}^{t}\left(\frac{H(t, s) p(s)}{r(s) \eta(s)}+h(t, s) \sqrt{H(t, s)}\right)^{2} \cdot \frac{r(s) \eta(s)}{H(t, s)} d s\right. \\
\left.+\sum_{k=1}^{N(t)} H\left(t, t_{k}\right) v\left(t_{k}\right) b_{k}\right\}=\infty,
\end{aligned}
$$

where $v=\exp \left\{-2 \int^{s} \frac{\rho(\tau)}{\xi(\tau)}-\frac{p}{2 r(\tau) \eta(\tau)} d \tau\right\}, \varphi(t)=-v(t)\left[(r(t) \rho(t))^{\prime}-q(t)+\frac{p(t) \rho(t)}{\zeta(t)}-\frac{r(t) \rho^{2}(t)}{\eta(t)}\right]$, then Eq. (1.2) is oscillatory.

Proof. Let $x(t)$ be a non-oscillatory solution of Eq. (1.2). We may assume that $x(t) \neq 0$ for all $t \geqslant T$. Consider the general Riccati transformation

$$
u_{1}(t)=v(t) r(t)\left[\Psi(x(t))\left(\frac{x^{\prime}(t)}{\chi(t)}\right)+\rho(t)\right]
$$

Then

$$
\begin{aligned}
u_{1}^{\prime}(t) & =\frac{v^{\prime}(t) u_{1}(t)}{v(t)}+v(t)\left[-p(t)\left(\frac{x^{\prime}(t)}{x(t)}\right)-q(t)-r(t) \Psi\left(x(t)\left(\frac{x^{\prime}(t)}{x(t)}\right)^{2}+(r(t) \rho(t))^{\prime}\right]\right. \\
& =\frac{v^{\prime}(t) u_{1}(t)}{v(t)}+v(t)\left[\frac{-p(t)}{\Psi(x(t)}\left(\frac{u_{1}(t)}{v(t) r(t)}-\rho(t)\right)-q(t)+(r(t) \rho(t))^{\prime}-r(t) \frac{1}{\Psi(x(t))}\left\{\frac{u_{1}(t)}{v(t) r(t)}-\rho(t)\right\}^{2}\right] .
\end{aligned}
$$

i.e.,

$$
u_{1}^{\prime}(t) \leqslant-\frac{u_{1}^{2}(t)}{\eta(t) v(t) r(t)}+v(t)\left[(r(t) \rho(t))^{\prime}-q(t)+\frac{p(t) \rho(t)}{\zeta(t)}-\frac{r(t) \rho^{2}(t)}{\eta(t)}\right]
$$

Thus

$$
u_{1}^{\prime}(t) \leqslant-\frac{u_{1}^{2}(t)}{\eta(t) v(t) r(t)}-\varphi(t), \text { for } t \neq t_{k} .
$$

Since $x(t)$ is continuous on $[T, \infty)$, then we have

$$
\begin{aligned}
\Delta u_{1}\left(t_{k}\right)=u_{1}\left(t_{k}^{+}\right)-u_{1}\left(t_{k}^{-}\right) & =\frac{r\left(t_{k}^{+}\right) \Psi\left(x\left(t_{k}^{+}\right)\right) x^{\prime}\left(t_{k}^{+}\right)}{x\left(t_{k}^{+}\right)}-\frac{r\left(t_{k}^{-}\right) \Psi\left(x\left(t_{k}^{-}\right)\right) x^{\prime}\left(t_{k}^{-}\right)}{x\left(t_{k}^{-}\right)} \\
& =\frac{r\left(t_{k}^{+}\right) \Psi\left(x\left(t_{k}^{+}\right)\right) x^{\prime}\left(t_{k}^{+}\right)-r\left(t_{k}^{-}\right) \Psi\left(x\left(t_{k}^{-}\right)\right) x^{\prime}\left(t_{k}^{-}\right)}{x\left(t_{k}\right)} \\
& =\frac{\Delta\left(r(t) \Psi(x) x^{\prime}\left(t_{k}\right)\right)}{x\left(t_{k}\right)}=-v\left(t_{k}\right) b_{k} .
\end{aligned}
$$

Therefore, the function $\mathfrak{u}_{1}(t)$ satisfies

$$
u_{1}^{\prime}(t) \leqslant-\frac{u_{1}^{2}(t)}{\eta(t) v(t) r(t)}-\varphi(t), \text { for } t \neq t_{k}, \quad \Delta u_{1}\left(t_{k}\right)+v\left(t_{k}\right) b_{k}=0 .
$$

Going through as in Theorem 2.1, we get

$$
\int_{T}^{t} \varphi(s) H(t, s) d s=H(t, T) u_{1}(T)-\int_{I} H(t, s)\left[\frac{u_{1}^{2}(s)}{r(s) \eta(s)}+p(s) \frac{u_{1}(s)}{r(s) \eta(s)}\right] d s
$$




$$
\left.-\sum_{k=1}^{N(t)} H\left(t, t_{k}\right) v\left(t_{k}\right) b_{k}-\int_{I} u_{1}(s) h(t, s) \sqrt{H(t, s}\right) d s
$$

Then

$$
\int_{T}^{t} \varphi(s) H(t, s) d s-\frac{1}{4} \int_{I}\left(\frac{H(t, s) p(s)}{r(s) \eta(s)}+h(t, s) \sqrt{H(t, s)}\right)^{2} \cdot \frac{r(s) \eta(s)}{H(t, s)} d s+\sum_{k=1}^{N(t)} H\left(t, t_{k}\right) b_{k} v\left(t_{k}\right) \leqslant H(t, T) u_{1}(T),
$$

i.e.,

$$
\begin{aligned}
& \int_{t_{0}}^{t} \varphi(s) H(t, s) d s-\frac{1}{4} \int_{t_{0}}^{t}\left(\frac{H(t, s) p(s)}{r(s) \eta(s)}+h(t, s) \sqrt{H(t, s)}\right)^{2} \cdot \frac{r(s) \eta(s)}{H(t, s)} d s+\sum_{k=1}^{N(t)} H\left(t, t_{k}\right) v\left(t_{k}\right) b_{k} \\
& \leqslant \int_{t_{0}}^{T} \varphi(s) H(t, s) d s+H(t, T) u_{1}(T)+\sum_{k=1}^{m-1} H\left(t, t_{k}\right) b_{k} v\left(t_{k}\right) \\
& \leqslant \int_{\mathrm{t}_{0}}^{\mathrm{T}}|\varphi(s)| \mathrm{H}(\mathrm{t}, \mathrm{s}) \mathrm{d} s+\mathrm{H}(\mathrm{t}, \mathrm{T})\left|\mathrm{u}_{1}(\mathrm{~T})\right|+\sum_{\mathrm{k}=1}^{\mathrm{m}-1} \mathrm{H}\left(\mathrm{t}, \mathrm{t}_{\mathrm{k}}\right)\left|\mathrm{b}_{\mathrm{k}} v\left(\mathrm{t}_{\mathrm{k}}\right)\right| \\
& \leqslant H\left(t, t_{0}\right)\left[\int_{t_{0}}^{T}|\varphi(s)| d s+\left|u_{1}(T)\right|+\sum_{k=1}^{m-1}\left|v\left(t_{k}\right) b_{k}\right|\right. \text {. }
\end{aligned}
$$

Therefore

$$
\begin{aligned}
\limsup _{t \rightarrow \infty} \frac{1}{H\left(t, t_{0}\right)} & \times\left\{\int_{t_{0}}^{t} \varphi(s) H(t, s)-\frac{1}{4} \int_{t_{0}}^{t}\left(\frac{H(t, s) p(s)}{r(s) \eta(s)}+h(t, s) \sqrt{H(t, s)}\right)^{2} \cdot \frac{r(s) \eta(s)}{H(t, s)} d s\right. \\
& \left.+\sum_{k=1}^{N(t)} H\left(t, t_{k}\right) v\left(t_{k}\right) b_{k}\right\}<\infty
\end{aligned}
$$

which contradicts (3.1), and completes the proof.

Remark 3.2.

1. In the special case $\Psi(x(t))=1$ and $p(t)=0$, the criterion (3.1) includes the condition (3.1) of [22]. Further in the special case $\Psi(x(t))=1, p>0$, and $r(t)=1$, the criterion (3.1) includes the condition (8) of [25].

2. In the case $H(t, s)=(t-s)^{\alpha}$ with $\alpha>1, h(t, s)=\alpha(t-s)^{\frac{\alpha-2}{2}}$, the functions $H(t, s)$ and $h(t, s)$ satisfy the conditions of Theorem 3.1, and Eq. (1.2) is oscillatory if

$$
\begin{aligned}
\limsup _{t \rightarrow \infty} \frac{1}{t^{\alpha}} & \times\left\{\int_{t_{0}}^{t} \varphi(s)(t-s)^{\alpha} d s+\sum_{k=1}^{N(t)}\left(t-t_{k}\right)^{\alpha} v\left(t_{k}\right) b_{k}\right. \\
& \left.-\int_{t_{0}}^{t} \frac{r(s) \eta(s)}{4}(t-s)^{\alpha-2}\left[\frac{(t-s) p(s)}{r(s) \eta(s)}+\alpha\right]^{2} d s\right\}=\infty,
\end{aligned}
$$

where $v(t)$ and $\varphi(t)$ are the same as in Theorem 3.1.

Theorem 3.3. For $\beta \geqslant 1$ and $\mathrm{H} \in \omega_{\gamma}$, if

$$
\begin{aligned}
& \limsup _{t \rightarrow \infty} \frac{1}{H\left(t, t_{0}\right)} \\
& \times\left\{\int_{t_{0}}^{t} H(t, s) \varphi_{1}(s) d s-\frac{\beta^{\gamma}}{(\gamma+1)^{\gamma+1}} v_{1}(s) r(s) \eta(s) h^{\gamma+1}(t, s) d s+\mu \sum_{k=1}^{N(t)} H\left(t, t_{k}\right) v_{1}\left(t_{k}\right) b_{k}\right\}=\infty,
\end{aligned}
$$


where

$$
v_{1}=\exp \left\{-(\gamma+1) \int_{\mathrm{t}_{0}}^{\mathrm{t}} \frac{\rho(s)}{\xi(s)}-\frac{p}{(\gamma+1) \mathrm{r}(\mathrm{t}) \eta(\mathrm{t})} \mathrm{ds}\right\}
$$

and

$$
\varphi_{1}(t)=-v_{1}(t)\left[(r(t) \rho(t))^{\prime}-\mu q(t)+\frac{p(t) \rho(t)}{\zeta(t)}-\frac{r(t) \rho^{1+\frac{1}{\gamma}}(t)}{\eta \frac{1}{\gamma}(t)}\right],
$$

then Eq. (1.3) is oscillatory.

Proof. Let $x(t)$ be a non-oscillatory solution of Eq. (1.3). Suppose that $x(t) \neq 0$ for all $t \geqslant T$. Let

$$
u_{2}(t)=v_{1}(t) r(t)\left[\Psi(x(t))\left(\frac{x^{\prime}(t)}{x(t)}\right)^{\gamma}+\rho(t)\right]
$$

Then

$$
\mathrm{u}_{2}^{\prime}(\mathrm{t})=\frac{v_{1}^{\prime}(\mathrm{t}) \mathrm{u}_{2}(\mathrm{t})}{v_{1}(\mathrm{t})}+v_{1}(\mathrm{t})\left[-\mathrm{p}(\mathrm{t})\left(\frac{\chi^{\prime}(\mathrm{t})}{\chi(\mathrm{t})}\right)^{\gamma}-\mathrm{q}(\mathrm{t}) \frac{\mathrm{f}(\mathrm{x})}{x^{\gamma}}-\gamma \frac{\mathrm{r}(\mathrm{t})}{\Psi(x(\mathrm{t}))^{\frac{1}{\gamma}}}\left(\frac{\mathrm{u}_{2}}{v_{1}(\mathrm{t}) \mathrm{r}(\mathrm{t})}-\rho(\mathrm{t})\right)^{\frac{\gamma+1}{\gamma}}+(\mathrm{r}(\mathrm{t}) \rho(\mathrm{t}))^{\prime}\right] .
$$

Using the first inequality of Lemma 1.2, we get

$$
\left(\frac{u_{2}}{v_{1}(t) r(t)}-\rho(t)\right)^{\frac{\gamma+1}{\gamma}} \leqslant \frac{\rho^{\frac{1}{\gamma}}(t)}{\gamma}\left[(\gamma+1)\left(\frac{u_{2}}{v_{1}(t) r(t)}-\rho(t)\right)\right]-\left(\frac{u_{2}}{v_{1}(t) r(t)}\right)^{1+\frac{1}{\gamma}} .
$$

Thus

$$
\begin{aligned}
\mathrm{u}_{2}^{\prime}(\mathrm{t})= & \frac{v_{1}^{\prime}(\mathrm{t}) \mathrm{u}_{2}(\mathrm{t})}{v_{1}(\mathrm{t})}+v_{1}(\mathrm{t})\left[-\mathrm{p}(\mathrm{t})\left(\frac{x^{\prime}(\mathrm{t})}{x(\mathrm{t})}\right)^{\gamma}-\mathrm{q}(\mathrm{t}) \frac{\mathrm{f}(\mathrm{x})}{x^{\gamma}}+(\mathrm{r}(\mathrm{t}) \rho(\mathrm{t}))^{\prime}\right. \\
& +\frac{\gamma \mathrm{r}(\mathrm{t})}{\Psi(x(\mathrm{t}))^{\frac{1}{\gamma}}} \frac{\rho^{\frac{1}{\gamma}}(\mathrm{t})}{\gamma}\left[(\gamma+1)\left(\frac{\mathrm{u}_{2}}{v_{1}(\mathrm{t}) \mathrm{r}(\mathrm{t})}-\rho(\mathrm{t})\right)\right]-\left(\frac{\mathrm{u}_{2}}{v_{1}(\mathrm{t}) \mathrm{r}(\mathrm{t})}\right)^{1+\frac{1}{\gamma}} .
\end{aligned}
$$

Substituting for $\Psi(x(t)), f(x)$ and using (3.4), we obtain

$$
u_{2}^{\prime}(t) \leqslant-\gamma\left[\frac{u_{2}^{\gamma+1}(t)}{\eta(t) v_{1}(t) r(t)}\right]^{\frac{1}{\gamma}}-\varphi_{1}(t), \text { for } t \neq t_{k}
$$

and

$$
\Delta u_{2}\left(t_{k}\right)=u_{2}\left(t_{k}^{+}\right)-u_{2}\left(t_{k}^{-}\right)=\frac{-v_{1}\left(t_{k}\right) b_{k} f(x)}{x^{\gamma}}=-\mu v_{1}\left(t_{k}\right) b_{k} .
$$

Using the same method in Theorem 3.1., we get

$$
\begin{aligned}
\int_{T}^{t} \varphi_{1}(s) H(t, s) d s \leqslant & H(t, T) u_{2}(T)-\mu \sum_{k=1}^{N(t)} H\left(t, t_{k}\right) v_{1}\left(t_{k}\right) b_{k}-\int_{I}\left[\gamma H(t, s)\left(\frac{u_{2}^{1+\gamma}(s)}{v_{1}(s) r(s) \eta(s)}\right)^{\frac{1}{\gamma}}\right. \\
& \left.-u_{2}(s) h(t, s) H^{\frac{\gamma}{\gamma+1}}(t, s)\right] d s .
\end{aligned}
$$

Thus for $\beta \geqslant 1$, we have

$$
\begin{gathered}
\int_{t_{0}}^{t}\left[\varphi_{1}(s) H(t, s)+u_{2}(s) h(t, s) H^{\frac{\gamma}{\gamma+1}}(t, s)+\frac{\gamma}{\beta} H(t, s)\left[\frac{u_{2}^{1+\gamma}(s)}{v_{1}(s) r(s) \eta(s)}\right]^{\frac{1}{\gamma}} d s+\mu \sum_{k=1}^{N(t)} H\left(t, t_{k}\right) v_{1}\left(t_{k}\right) b_{k}\right. \\
\leqslant H(t, T) u_{2}(T)-\frac{\gamma}{\beta}(\beta-1) \int_{t_{0}}^{t} H(t, s)\left[\frac{u_{2}^{1+\gamma}(s)}{v_{1}(s) r(s) \eta(s)}\right]^{\frac{1}{\gamma}} d s .
\end{gathered}
$$


Applying the second inequality of Lemma 1.2, we get

$$
\begin{aligned}
& \int_{t_{0}}^{t}\left[\varphi_{1}(s) H(t, s)-\right.\left.\frac{\beta^{\gamma} v_{1}(s) r(s) \eta(s) h \gamma+1}{(t, s)}\right] d s+\mu \sum_{k=1}^{N(t)} H\left(t, t_{k}\right) v_{1}\left(t_{k}\right) b_{k} \\
&\left.\leqslant H(t, T) u_{2}(T)-\frac{\gamma}{\beta}(\beta-1) \int_{t_{0}}^{t} H(t, s)\left[\frac{u_{2}^{1+\gamma}(s)}{v_{1}(s) r(s) \eta(s)}\right] \frac{1}{\gamma}\right] d s .
\end{aligned}
$$

Using the properties of $\mathrm{H}(\mathrm{t}, \mathrm{s})$, we have

$$
\begin{aligned}
\int_{t_{0}}^{t}\left[\varphi_{1}(s) H(t, s)-\right. & \left.\frac{\beta^{\gamma} v_{1}(s) r(s) \eta(s) h \gamma+1(t, s)}{(\gamma+1)^{\gamma+1}}\right] d s+\mu \sum_{k=1}^{N(t)} H\left(t, t_{k}\right) v_{1}\left(t_{k}\right) b_{k} \\
& \leqslant H(t, T)\left|u_{2}(T)\right| \int_{t_{0}}^{T}|\varphi(s)| H(t, s) d s++\mu \sum_{k=1}^{m-1} H\left(t, t_{k}\right)\left|b_{k} v\left(t_{k}\right)\right| \\
& \leqslant H\left(t, t_{0}\right)\left[\int_{t_{0}}^{T}|\varphi(s)| d s+\left|u_{2}(T)\right|+\sum_{k=1}^{m-1}\left|v\left(t_{k}\right) b_{k}\right| .\right.
\end{aligned}
$$

Therefore

$$
\limsup _{t \rightarrow \infty} \frac{1}{H\left(t, t_{0}\right)}\left\{\int_{t_{0}}^{t} H(t, s) \varphi_{1}(s) d s-\frac{\beta^{\gamma}}{(\gamma+1)^{\gamma+1}} v_{1}(s) r(s) \eta(s) h^{\gamma+1}(t, s) d s+\mu \sum_{k=1}^{N(t)} H\left(t, t_{k}\right) v_{1}\left(t_{k}\right) b_{k}\right\}<\infty,
$$

which contradicts (3.2). The case $x<0$, is similar and this completes the proof.

Remark 3.4. For $b_{k}=0$ (the case of no impulse effect) and $\Psi(x(t))=1$, Eq. (1.3) takes the form

$$
\left[r(t)\left(x^{\prime}(t)\right)^{\gamma}\right]^{\prime}+p(t)\left(x^{\prime}(t)\right)^{\gamma}+q(t) f(x(t))=0,
$$

which was studied by Li et al. [18]. The criterion (3.3) improves and extends Theorem 2 of [18] but in the presence of impulse term.

\section{Examples}

Example 4.1. Consider the differential equations

$$
\left[\Psi(x(t)) x^{\prime}(t)\right]^{\prime}+\epsilon x(t)=0, t \geqslant t_{0}, t \neq k ; \quad \Delta\left[\Psi(x(t)) x^{\prime}(t)\right]+\varpi x(k)=0,
$$

where $\Psi(x(t))$ is any function satisfying the condition $t \leqslant \Psi(x(t)) \leqslant 2 t, k \in N$ and $\epsilon, \varpi>0$. Here, $r(t)=1, p(t)=0, q(t)=\epsilon, t_{k}=k$ and $b_{k}=\varpi$ for all $k \in N$. Choosing $\rho=\frac{1}{2}$ and $\alpha=2$, then

$$
v=\exp \left\{-2 \int_{t_{0}}^{t} \frac{\rho(s)}{\xi(s)}-\frac{p}{2 r(t) \eta(t)} d s\right\}=\exp \{-\ln t\}=\frac{1}{t},
$$

and

$$
\varphi(t)=-v(t)\left[(r(t) \rho(t))^{\prime}-q(t)+\frac{p(t) \rho(t)}{\zeta(t)}-\frac{r(t) \rho^{2}(t)}{\eta(t)}\right]=\frac{\epsilon}{t}+\frac{1}{8 t^{2}} .
$$

So we have for $t \geqslant t_{0}$,

$$
\frac{1}{\mathrm{t}^{2}} \times \int_{\mathrm{t}_{0}}^{\mathrm{t}} \varphi(\mathrm{s})(\mathrm{t}-\mathrm{s})^{2}-\mathrm{r}(\mathrm{s}) \eta(\mathrm{s}) \mathrm{d} s=\frac{1}{\mathrm{t}^{2}} \times \int_{\mathrm{t}_{0}}^{\mathrm{t}}\left\{\left[\frac{\epsilon}{\mathrm{s}}+\frac{1}{8 \mathrm{~s}^{2}}\right]\left[\mathrm{t}^{2}-2 \mathrm{st}+\mathrm{s}^{2}\right]-2 \mathrm{~s}\right\} \mathrm{d} s
$$




$$
\begin{aligned}
& =\int_{\mathrm{t}_{0}}^{\mathrm{t}}\left[\frac{\epsilon}{\mathrm{s}}+\frac{1}{8 \mathrm{~s}^{2}}\right]\left[1-\frac{2 \mathrm{~s}}{\mathrm{t}}+\frac{\mathrm{s}^{2}}{\mathrm{t}^{2}}\right]-\frac{2 \mathrm{~s}}{\mathrm{t}^{2}} \mathrm{ds} \\
& =\int_{\mathrm{t}_{0}}^{\mathrm{t}}\left[\frac{\epsilon}{\mathrm{s}}+\frac{1}{8 \mathrm{~s}^{2}}\right]-2\left[\frac{\epsilon}{\mathrm{t}}+\frac{1}{s \mathrm{t}}\right]+\left[\frac{\epsilon}{s \mathrm{t}^{2}}+\frac{1}{8 \mathrm{t}^{2}}\right]-\frac{2 \mathrm{~s}}{\mathrm{t}^{2}} \mathrm{~d} s \\
& =\left\{\epsilon \ln s-\frac{1}{8 \mathrm{~s}}-2\left[\frac{\epsilon s}{\mathrm{t}}+\frac{\ln \mathrm{s}}{\mathrm{t}}\right]+\frac{\epsilon}{\mathrm{t}^{2}} \ln \mathrm{s}+\frac{\mathrm{s}}{8 \mathrm{t}^{2}}-\frac{\mathrm{s}^{2}}{\mathrm{t}^{2}}\right\}_{\mathrm{t}_{0}}^{\mathrm{t}} .
\end{aligned}
$$

Then

$$
\limsup _{t \rightarrow \infty} \frac{1}{t^{2}} \times\left\{\int_{t_{0}}^{t} \varphi(s)(t-s)^{2}-r(s) \eta(s) d s\right\}<\infty .
$$

Now, to examine the series term

$$
\limsup _{t \rightarrow \infty} \frac{1}{t^{2}} \sum_{k=1}^{m}\left(t-t_{k}\right)^{2} v\left(t_{k}\right) b_{k}=\limsup _{t \rightarrow \infty} \frac{1}{t^{2}} \sum_{k=1}^{m} \frac{\varpi}{k}(t-k)^{2}>\limsup _{t \rightarrow \infty} \sum_{k=1}^{m} \frac{\varpi}{k}-\sum_{k=1}^{m} \frac{2 \varpi}{t}+\sum_{k=1}^{m} \frac{\varpi k}{t^{2}} .
$$

Since

$$
\sum_{k=1}^{m} \frac{\varpi}{k}=\varpi \sum_{k=1}^{m} \frac{1}{k}>\varpi \int_{1}^{m+1} \frac{1}{k} d s=\ln (m+1), \quad \sum_{k=1}^{m} \frac{2 \varpi}{t}=\frac{2 \varpi}{t} \sum_{k=1}^{m} 1=2 \varpi,
$$

and

$$
\sum_{k=1}^{m} \frac{\varpi k}{t^{2}}=\frac{\varpi}{(m+1)^{2}} \sum_{k=1}^{m} k=\frac{\varpi}{(m+1)^{2}} \times \frac{m(m+1)}{2}=\frac{m \varpi}{2(m+1)}
$$

we get

$$
\limsup _{t \rightarrow \infty} \frac{1}{t^{2}} \sum_{k=1}^{m}\left(t-t_{k}\right)^{2} v\left(t_{k}\right) b_{k}=\limsup _{t \rightarrow \infty}\left\{\ln (m+1)-2 \varpi+\frac{m \varpi}{2(m+1)}\right\}=\infty .
$$

So Eq. (4.1) is oscillatory.

Example 4.2. Consider the differential equations

$$
\left[\frac{1}{t} \Psi(x(t))\left(x^{\prime}(t)\right)^{\gamma}\right]^{\prime}+\frac{A}{t^{2}} x^{\gamma}(t)=0, t \geqslant t_{0}, t \neq k ; \quad \Delta\left[\frac{1}{t} \Psi(x(t))\left(x^{\prime}(t)\right)^{\gamma}\right]+\frac{B}{k} x^{\gamma}(k)=0,
$$

where $\Psi(x(t))$ is any function satisfying the condition $t \leqslant \Psi(x(t)) \leqslant(\gamma+1) t, k \in N$ and $A, B>0$. Here, $r(t)=\frac{1}{t}, p(t)=0, q(t)=\frac{A}{t^{2}}, t_{k}=k, f(x)=x^{\gamma}, \mu=1$ and $b_{k}=\frac{B}{k}$ for all $k \in N$. Choosing $\rho=0$ and $H(t, s)=(t-s)^{2}, \beta=\frac{(\gamma+1)^{1+\frac{1}{\gamma}}}{2}$, then

$$
\begin{gathered}
v_{1}=\exp \left\{-(\gamma+1) \int_{\mathrm{t}_{0}}^{\mathrm{t}} \frac{\rho(s)}{\xi(s)}-\frac{p}{(\gamma+1) r(t) \eta(t)} \mathrm{ds}\right\}=1, \\
\varphi_{1}(\mathrm{t})=-v_{1}(\mathrm{t})\left[(\mathrm{r}(\mathrm{t}) \rho(\mathrm{t}))^{\prime}-\mu \mathrm{q}(\mathrm{t})+\frac{p(\mathrm{t}) \rho(\mathrm{t})}{\zeta(\mathrm{t})}-\frac{\mathrm{r}(\mathrm{t}) \rho^{1+\frac{1}{\gamma}}(\mathrm{t})}{\eta \frac{1}{\gamma}(\mathrm{t})}\right],
\end{gathered}
$$

and

$$
h^{\gamma+1}=2^{\gamma+1}(t-s)^{(1-\gamma)} .
$$

Then for $t \geqslant t_{0}$, we get

$$
\begin{aligned}
\limsup _{t \rightarrow \infty} \frac{1}{H\left(t, t_{0}\right)} & \times\left\{\int_{t_{0}}^{t} H(t, s) \varphi_{1}(s) d s-\frac{\beta^{\gamma}}{(\gamma+1)^{\gamma+1}} v_{1}(s) r(s) \eta(s) h^{\gamma+1}(t, s) d s\right\} \\
& =\limsup _{t \rightarrow \infty} \frac{1}{t^{2}} \times\left\{\int_{t_{0}}^{t} \varphi_{1}(s)(t-s)^{2}-\frac{\beta^{\gamma}}{(\gamma+1)^{\gamma+1}} v_{1} r(s) \eta(s) h^{\gamma+1}(t, s) d s\right\} \\
& =\limsup _{t \rightarrow \infty} \frac{1}{t^{2}} \times \int_{t_{0}}^{t}\left\{\left[\frac{A}{s^{2}}\right]\left[t^{2}-2 s t+s^{2}\right]-2(\gamma+1)(t-s)^{1-\gamma}\right\} d s \\
& \geqslant \limsup _{t \rightarrow \infty} \frac{1}{t^{2}} \times \int_{t_{0}}^{t}\left\{\left[\frac{A}{s^{2}}\right]\left[t^{2}-2 s t+s^{2}\right]-2(\gamma+1)\right\} d s=\frac{A}{t_{0}}<\infty .
\end{aligned}
$$


Now, to examine the series term

$$
\begin{aligned}
\limsup _{t \rightarrow \infty} \frac{\mu}{t^{2}} \sum_{k=1}^{N(t)} H\left(t, t_{k}\right) v_{1}\left(t_{k}\right) b_{k} & =\limsup _{t \rightarrow \infty} \frac{\mu}{t^{2}} \sum_{k=1}^{m}\left(t-t_{k}\right)^{2} v_{1}\left(t_{k}\right) b_{k} \\
& =\limsup _{t \rightarrow \infty} \frac{1}{t^{2}} \sum_{k=1}^{m} \frac{B}{k}(t-k)^{2}>\limsup _{t \rightarrow \infty} \sum_{k=1}^{m} \frac{B}{k}-\sum_{k=1}^{m} \frac{2 B}{t}+\sum_{k=1}^{m} \frac{B k}{t^{2}} .
\end{aligned}
$$

Since

$$
\sum_{k=1}^{m} \frac{B}{k}=B \sum_{k=1}^{m} \frac{1}{k}>B \int_{1}^{m+1} \frac{1}{k} d s=\ln (m+1), \quad \sum_{k=1}^{m} \frac{2 B}{t}=\frac{2 B}{t} \sum_{k=1}^{m} 1=2 B,
$$

and

$$
\sum_{k=1}^{m} \frac{B k}{t^{2}}=\frac{B}{(m+1)^{2}} \sum_{k=1}^{m} k=\frac{B}{(m+1)^{2}} \times \frac{m(m+1)}{2}=\frac{m B}{2(m+1)^{\prime}}
$$

we have

$$
\limsup _{t \rightarrow \infty} \frac{\mu}{t^{2}} \sum_{k=1}^{m}\left(t-t_{k}\right)^{2} v_{1}\left(t_{k}\right) b_{k}=\limsup _{t \rightarrow \infty}\left\{\ln (m+1)-2 B+\frac{m B}{2(m+1)}\right\}=\infty .
$$

Therefore, condition (3.2) holds at $\alpha=2$. Then the conditions of Theorem 3.3 are satisfied, and Eq. (4.2) is oscillatory.

Remark 4.3. We may note that, the oscillation of Eq. (4.2) is caused by the effect of the impulses, and it is non-oscillatory at $b_{k}=0$.

\section{Acknowledgment}

The authors thank the anonymous reviewers for their important and valuable comments.

\section{References}

[1] U. A. Abasiekwere, I. M. Esuabana, I. O. Isaac, Z. Lipscey, Oscillations of second order impulsive differential equations with advanced arguments, Global J. Sci. Frontier Res. Math. Decis. Sci. (USA), 18 (2018), 25-32. 1

[2] H. K. Abdullah, A note on the oscillation of the second order differential equations, Czechoslovak Math. J., 54 (2004), 949-954. 1

[3] R. P. Agarwal, M. Bohner, T. X. Li, Oscillatory behavior of second-order half-linear damped dynamic equations, Appl. Math. Comput., 254 (2015), 408-418. 1

[4] R. P. Agarwal, M. Bohner, T. X. Li, C. H. Zhang, Oscillation criteria for second-order dynamic equations on time scales, Appl. Math. Lett., 31 (2014), 34-40. 1

[5] R. P. Agarwal, F. Karakoç, A survey on oscillation of impulsive delay differential equations, Comput. Math. Appl., 60 (2010), 1648-1648. 1

[6] R. P. Agarwal, F. Karakoç, A. Zafer, A survey on oscillation of impulsive differential equations, Adv. Difference Equ., 2010 (2010), 52 pages. 1

[7] R. P. Agarwal, C. H. Zhang, T. X. Li, New Kamenev-type oscillation criteria for second-order nonlinear advanced dynamic equations, Appl. Math. Comput., 225 (2013), 822-828. 1

[8] D. D. Bainov, P. Simeonov, Oscillation theory of impulsive differential equations, International Publications, Orlando, (1998).

[9] M. Bohner, T. S. Hassan, T. X. Li, Fite-Hille-Wintner-type oscillation criteria for second-order half-linear dynamic equation with deviating arguments, Indag. Math. (N.S.), 29 (2018), 548-560.

[10] M. Bohner, T. X. Li, Kamenev-type criteria for nonlinear damped dynamic equations, Sci. China Math., 58 (2015), 14451452.

[11] J. Džurina, S. R. Grace, I. Jadlovská, T. X. Li, Oscillation criteria for second-order Emden-Fowler delay differential equations with a sublinear neutral term, Math. Nachr., 293 (2020), 910-922.

[12] I. M. Esuabana, U. A. Abasiekwere, On stability of first order linear impulsive differential equations, Int. J. Stat. Appl. Math., 3 (2018), 231-236. 1 
[13] Z. M. He, W. G. Ge, Oscillation of second order nonlinear impulsive ordinary differential equations, J. Comput. Appl. Math., 158 (2003), 397-406. 1

[14] V. Lakshmikantham, D. D. Bainov, P. S. Simeonov, Theory of Impulsive Differential Equations, World Scientific Publishing Co., Teaneck, (1989). 1

[15] J. H. Li, Oscillation criteria for second order linear differential equations, J. Math. Anal. Appl., 194 (1995), $217-234.1$

[16] T. X. Li, N. Pintus, G. Viglialoro, Properties of solutions to porous medium problems with different sources and boundary conditions, Z. Angew. Math. Phys., 70 (2019), 18 pages. 1

[17] T. X. Li, Y. V. Rogovchenko, Oscillation criteria for second-order superlinear Emden-Fowler neutral differential equations, Monatsh. Math., 184 (2017), 489-500. 1

[18] T. X. Li, Y. V. Rogovchenko, S. H. Tang, Oscillation of second-order nonlinear differential equations with damping, Math. Slovaca, 64 (2014), 1227-1236. 1.2, 3.4

[19] T. X. Li, S. H. Saker, A note on oscillation criteria for second-order neutral dynamic equations on isolated time scales, Commun. Nonlinear Sci. Numer. Simul., 19 (2014), 4185-4188. 1

[20] T. Li, G. Viglialoro, Boundedness for a nonlocal reaction chemotaxis model even in the attraction dominated regime, Differ. Integ. Equ., 34 (2021), 315-336. 1

[21] Y. V. Rogovchenko, Oscillation theorems for second order equations with damping, Nonlinear Anal., 41 (2000), 10051028. 1

[22] J. Sugie, Interval criteria for oscillation of second order self-adjoint impulsive differential equations, Proc. Amer. Math. Soc., 148 (2019), 1095-1108. 1, 1, 2, 2.2, 2.4, 1

[23] J. Sugie, K. Ishihara, Philos-type oscillation criteria for linear differential equations with impulsive effects, J. Math. Anal. Appl., 470 (2019), 911-930. 1

[24] G. Viglialoro, On the blow-up time of a parabolic system with damping terms, C. R. Acad. Bulgare Sci., 67 (2014), 1223-1232. 1

[25] K. W. Wen, Y. P. Zeng, H. Q. Peng, L. F. Huang, Philos-type oscillation criteria for second-order linear impulsive differential equation with damping, Bound. Value Probl., 111 (2019), 16 pages. 1, 1, 1.1, 2, 2.2, 2, 2.4, 1

[26] D. Willett, On the oscillatory behavior of the solutions of second order differential equations, Ann. Polon Math., 21 (1969), 175-194. 1

[27] J. R. Yan, A note on an oscillation criterion for an equation with damped term, Proc. Amer. Math. Soc., 90 (1984), $277-280$. 1

[28] C. H. Zhang, R. P. Agarwal, T. X. Li, Oscillation and asymptotic behavior of higher-order delay differential equations with p-Laplacian like operators, J. Math. Anal. Appl., 409 (2014), 1093-1106. 1 